\title{
Isometric composition operators on weighted Dirichlet-type spaces
}

\author{
Li-Gang Geng ${ }^{1}$, Ze-Hua Zhou ${ }^{2 *}$ and Xing-Tang Dong ${ }^{2}$
}

* Correspondence:

zehuazhou2003@yahoo.com.cn

${ }^{2}$ Department of Mathematics,

Tianjin University, Tianjin 300072,

People's Republic of China

Full list of author information is

available at the end of the article

\section{Abstract}

In this article, we characterize the surjective isometric composition operator $C_{\phi}$ on the weighted Dirichlet-type spaces of the unit disk $\mathbb{D}$, where $\phi$ is an analytic selfmap of $\mathbb{D}$, and show that $C_{\phi}$ is a surjective isometry if and only if $\phi$ is a rotation map.

2000 MSC: Primary: 47B33; Secondary: 30D45; 30H30; 30H05; 47B38.

Keywords: rotation map, composition operator, weighted Dirichlet-type spaces, isometry

\section{Introduction}

The Lebesgue area measure on the unit disk $\mathbb{D}$ in the complex plane is defined by $d A$ $(z)=r d r d t=d x d y$. Denote by $H(\mathbb{D})$ the class of all analytic functions on $\mathbb{D}$ and $S(\mathbb{D})$ the collection of all the analytic self mappings of $\mathbb{D}$.

Given real numbers $p>0$ and $\alpha>-1$, the weighted Bergman space $A_{\alpha}^{p}$ is defined as the space of $f$ in $H(\mathbb{D})$ such that

$$
\|f\|_{A_{\alpha}^{p}}=\left(\int_{\mathbb{D}}|f(z)|^{p}\left(1-|z|^{2}\right)^{\alpha}(\alpha+1) d A\right)^{1 / p}<\infty .
$$

The weighted Dirichlet-type space $\mathcal{D}_{\alpha}^{p}$ is the space of $f$ in $H(\mathbb{D})$ such that $f^{\prime} \in A_{\alpha}^{p}$, equipped with the "norm":

$$
\|f\|_{\mathcal{D}_{\alpha}^{p}}=|f(0)|+\left\|f^{\prime}\right\|_{A_{\alpha}^{p}} .
$$

It is not a true norm for $0<p<1$, but it satisfies

$$
\|f+g\|_{\mathcal{D}_{\alpha}^{p}} \leq C_{p}\left(\|f\|_{\mathcal{D}_{\alpha}^{p}}+\|g\|_{\mathcal{D}_{\alpha}^{p}}\right)
$$

where the constant $C_{p}$ depends only on $p$. We write $\mathcal{D}^{p}=\mathcal{D}_{0}^{p}$. The space $\mathcal{D}=\mathcal{D}^{2}$ is the classical Dirichlet space of analytic functions whose image Riemann surface has finite area. Clearly, $\mathcal{D}^{p} \subset \mathcal{D}^{q}$ when $q<p<\infty$.

For $u \in H(\mathbb{D})$ and $\varphi \in S(\mathbb{D})$, the composition operator $C_{\phi}$ induced by $\phi$ is defined as $C_{\phi} f=f \circ \phi$ for $f \in H(\mathbb{D})$; the multiplication operator $M_{u}$ induced by $u$ is defined by $M_{u} f(z)=u(z) f(z)$; and the weighted composition operator $W_{u, \phi}$ induced by $\phi$ and $u$ is defined by $\left(W_{u, \phi} f\right)(z)=u(z) f(\phi(z))$ for $z \in \Omega$ and $f \in H(\Omega)$. If we let $u \equiv 1$, then 
$W_{u, \phi}=C_{\phi}$; if we let $\phi=i d$, then $W_{u, \phi}=M_{u}$. So we can regard weighted composition operator as a generalization of a multiplication operator and a composition operator. These operators are linear. In [1], Hibschweiler studied the boundedness and compactness of composition operators on $\mathcal{D}^{p}$. In [2], Roan studied the boundness of composition operators on $S^{p}$, where $S^{p}=\left\{f \in H(\mathbb{D}): f^{\prime} \in H^{p}\right\}$.

An operator $T$ on a normed space $\mathbf{X}$ is said to be an isometric operator if $\|T f\|_{\mathbf{X}}=\|$ $\left.f\right|_{\mathbf{X}}$, for any $f \in \mathbf{X}$.

The isometric composition operator on analytic functions spaces has been studied by many authors. In [3], Martín and Vukotić studied the isometric composition operators on $H^{p}, A_{\alpha}^{p}$ (see also Kolaski $[4,5]$ ) for $1 \leq p<\infty$ and the analytic Besov spaces $B^{p}$ for 1 $<p<\infty$. They obtained that $C_{\phi}$ is an isometry of $H^{p}$ if and only if $\phi$ is inner and $\phi(0)$ $=0$, and $C_{\phi}$ is an isometry of $A_{\alpha}^{p}$ if and only if $\phi$ is a rotation. Also Carswell and Hammond [6] obtained that $C_{\varphi}: A_{\alpha}^{2} \rightarrow A_{\alpha}^{2}$ is an isometry if and only if $\phi$ is a rotation; this fact differs somewhat from the analogs results that are known for other Hilbert spaces.

The isometric composition operators on the Bloch spaces in the unit disk were discussed by Martín and Vukotić [7], Colonna [8], Allen and Colonna [9,10], Li and Zhou [11]. The same problems were studied on the Bloch spaces in the unit polydisk by Cohen and Colonna [12], in unit ball by Li [13], and Li and Ruan [14]. For the BMOA space, see [15]. In [16], Martín and Vukotić also studied the isometric composition operators on the classical Dirichlet spaces in the unit disk. They obtained that $C_{\phi}$ is an isometric operator if and only if $\phi$ is a univalent map of the unit disk such that $A[\mathbb{D} \backslash \varphi(\mathbb{D})]=0$ and $\phi(0)=0$. In [17], Novinger and Oberlin studied the isometric composition operators on $S^{p}$ with different norms.

The present article continues this line of research and discusses the isometric composition operators on the weighted Dirichlet-type space in the unit disk.

\section{Main results}

Our proofs will depend upon a characterization of the linear isometries of weighted Bergman spaces due to Kolaski [4]. Although not stated by Kolaski explicitly, the following result is a direct consequence of Theorems 1 and 4 in [4], which are much more general results than we will need here.

Theorem 2.1. Let $0<p<\infty, p \neq 2$, and $\alpha>-1$. Then every linear isometry $T: A_{\alpha}^{p} \rightarrow A_{\alpha}^{p}$ takes the form $(T f)(z)=g(z) \cdot f(\phi(z))$, for all $f \in A_{\alpha}^{p}$ and $z \in \mathbb{D}$, for some function $\phi$ which maps $\mathbb{D}$ conformally onto a dense subset of $\mathbb{D}$, and where $g=T 1$. Moreover, if $T$ is surjective, then $\phi$ is a disk automorphism and $g=\lambda\left(\phi^{\prime}\right)^{(2+\alpha) / p}$ for some $|\lambda|=1$.

Following the ideas of Theorem 4.5.1 in [18], we investigate the surjective isometric composition operators on the weighted Dirichlet-type space.

Theorem 2.2. Let $\phi$ be a self-map of the unit disk. Then the induced composition operator $C_{\phi}$ is a surjective isometry on the weighted Dirichlet-type space $\mathcal{D}_{\alpha}^{p}, 1 \leq p<$ $\infty, p \neq 2,-1<\alpha<\infty$ if and only if $\phi$ is a rotation map.

Proof. Since the composition operator induced by a rotation is clearly an isometry, it suffices to show that if $C_{\phi}$ is a surjective isometry, then $\phi$ is a rotation.

Suppose that $C_{\phi}$ is a surjective isometric composition operator. Let $n$ be a positive integer and $t$ be a real number. We define the function $p_{n}(z)=z^{n}$ for all $z \in \mathbb{D}$. The 
weighted Dirichlet-type space contains the polynomials and thus $1+t p_{n} \in \mathcal{D}_{\alpha}^{p}$ for every real number $t$ and positive integer $n$.

Using the definition of norm of $\mathcal{D}_{\alpha}^{p}$ and that $C_{\phi}$ is an isometry, we have

$$
\left\|C_{\varphi} 1\right\|_{\mathcal{D}_{\alpha}^{p}}=\|1\|_{\mathcal{D}_{\alpha}^{p}}, \quad\left\|C_{\varphi} p_{n}\right\|_{\mathcal{D}_{\alpha}^{p}}=\left\|p_{n}\right\|_{\mathcal{D}_{\alpha}^{p}}
$$

and

$$
\left\|C_{\varphi}\left(1+t p_{n}\right)\right\|_{\mathcal{D}_{\alpha}^{p}}=\left\|1+t p_{n}\right\|_{\mathcal{D}_{\alpha}^{0}} .
$$

It follows that

$$
\begin{aligned}
\left\|1+t p_{n}\right\|_{\mathcal{D}_{\alpha}^{p}} & =1+\left(\int_{\mathbb{D}}\left|\left(t p_{n}\right)^{\prime}\right|^{p}\left(1-|z|^{2}\right)^{\alpha}(\alpha+1) d A\right)^{\frac{1}{p}} \\
& =\|1\|_{\mathcal{D}_{\alpha}^{p}}+\left\|t p_{n}\right\|_{\mathcal{D}_{\alpha}^{p}}=\|1\|_{\mathcal{D}_{\alpha}^{p}}+|t|\left\|p_{n}\right\|_{\mathcal{D}_{\alpha}^{p}} \\
& =\left\|C_{\varphi} 1\right\|_{\mathcal{D}_{\alpha}^{p}}+|t|\left\|C_{\varphi} p_{n}\right\|_{\mathcal{D}_{\alpha}^{p}} \\
& =\left|C_{\varphi} 1(0)\right|+|t|\left|C_{\varphi} p_{n}(0)\right|+\left\|\left(C_{\varphi} 1\right)^{\prime}\right\|_{A_{\alpha}^{p}}+|t|\left\|\left(C_{\varphi} p_{n}\right)^{\prime}\right\|_{A_{\alpha}^{p}}
\end{aligned}
$$

and

$$
\left\|C_{\varphi}\left(1+t p_{n}\right)\right\|_{\mathcal{D}_{\alpha}^{p}}=\left|C_{\varphi} 1(0)+t C_{\varphi} p_{n}(0)\right|+\left\|\left(C_{\varphi} 1\right)^{\prime}+t\left(C_{\varphi} p_{n}\right)^{\prime}\right\|_{A_{\alpha}^{p}} .
$$

So

$$
\begin{aligned}
& \left|C_{\varphi} 1(0)+t C_{\varphi} p_{n}(0)\right|+\left\|\left(C_{\varphi} 1\right)^{\prime}+t\left(C_{\varphi} p_{n}\right)^{\prime}\right\|_{A_{\alpha}^{p}} \\
& =\left|C_{\varphi} 1(0)\right|+|t|\left|C_{\varphi} p_{n}(0)\right|+\left\|\left(C_{\varphi} 1\right)^{\prime}\right\|_{A_{\alpha}^{p}}+|t|\left\|\left(C_{\varphi} p_{n}\right)^{\prime}\right\|_{A_{\alpha}^{p} .}
\end{aligned}
$$

A simple application of the triangle inequality for the norms shows that

$$
\left|C_{\varphi} 1(0)+t C_{\varphi} p_{n}(0)\right| \leq\left|C_{\varphi} 1(0)\right|+|t|\left|C_{\varphi} p_{n}(0)\right|
$$

and

$$
\left\|\left(C_{\varphi} 1\right)^{\prime}+t\left(C_{\varphi} p_{n}\right)^{\prime}\right\|_{A_{\alpha}^{p}} \leq\left\|\left(C_{\varphi} 1\right)^{\prime}\right\|_{A_{\alpha}^{p}}+|t|\left\|\left(C_{\varphi} p_{n}\right)^{\prime}\right\|_{A_{\alpha}^{p}} .
$$

Consequently,

$$
\left|C_{\varphi} 1(0)+t C_{\varphi} p_{n}(0)\right|=\left|C_{\varphi} 1(0)\right|+|t|\left|C_{\varphi} p_{n}(0)\right|
$$

and

$$
\left\|\left(C_{\varphi} 1\right)^{\prime}+t\left(C_{\varphi} p_{h}\right)^{\prime}\right\|_{A_{\alpha}^{p}}=\left\|\left(C_{\varphi} 1\right)^{\prime}\right\|_{A_{\alpha}^{p}}+|t|\left\|\left(C_{\varphi} p_{h}\right)^{\prime}\right\|_{A_{\alpha}^{p}} .
$$

In particular, for $n=1$, we get $|1+t \phi(0)|=1+|t||\phi(0)|$ which implies, since $t$ is an arbitrary real number, that $\phi(0)=0$.

Therefore $\left\|C_{\varphi} f\right\|_{\mathcal{D}_{\alpha}^{p}}=\|f\|_{\mathcal{D}_{\alpha}^{p}}$ is equivalent to $\left\|\left(C_{\varphi} f\right)^{\prime}\right\|_{A_{\alpha}^{p}}=\left\|f^{\prime}\right\|_{A_{\alpha}^{p}}$.

Let $\mathcal{D}_{\alpha, 0}^{p}$ denotes the subspace of functions in $\mathcal{D}_{\alpha}^{p}$ that vanish at the origin. The differentiation operator $D$ maps $\mathcal{D}_{\alpha, 0}^{p}$ isometrically onto $A_{\alpha}^{p}$ and its inverse $I$ is given by

$$
I g(z)=\int_{0}^{z} g(\xi) d \xi
$$


and maps $A_{\alpha}^{p}$ isometrically onto $\mathcal{D}_{\alpha, 0}^{p}$.

Since $C_{\phi}$ is a surjective isometry on $\mathcal{D}_{\alpha}^{p}$, for every $f \in \mathcal{D}_{\alpha, 0}^{p}$, there exists $g \in \mathcal{D}_{\alpha}^{p}$ such that $C_{\phi} g=f$. Because

$$
0=f(0)=\left(C_{\varphi} g\right)(0)=g(\varphi(0))=g(0),
$$

then $g \in \mathcal{D}_{\alpha}^{p}$. Thus the isometric composition operator $C_{\phi}$ maps the subspace $\mathcal{D}_{\alpha, 0}^{p}$ onto itself. So the composition $D C_{\varphi} I: A_{\alpha}^{p} \rightarrow A_{\alpha}^{p}$ is a surjective isometric operator. Set $T=D C_{\phi} I$. Then by Theorem 2.1, there exists an automorphism $\phi$ of $\mathbb{D}$ such that $T f(z)$ $=g(z) f(\phi(z))$, for all $f \in A_{\alpha}^{p}, z \in \mathbb{D}$, where $g=T 1$. Then, noting that $I(f)=f-f(0)$, we obtain

$$
g(z) f^{\prime}(\phi(z))=T f^{\prime}(z)=D C_{\varphi} I f^{\prime}(z)=D C_{\varphi}(f(z)-f(0))=f^{\prime}(\varphi(z)) \varphi^{\prime}(z) .
$$

Therefore,

$$
f^{\prime}(\varphi(z)) \varphi^{\prime}(z)=g(z) f^{\prime}(\phi(z))
$$

for any $f \in \mathcal{D}_{\alpha}^{p}$.

Letting $f=i d$ in (2.1), then $\phi^{\prime}(z)=g(z)$ (Alternatively, one can see that $\phi^{\prime}=g$ since $g$ $\left.(z)=(T 1)(z)=D C_{\phi} I_{1}(z)=\phi^{\prime}(z)\right)$. Letting $f=p_{2}$, then $\phi(z) \phi^{\prime}(z)=g(z) \phi(z)$, so from $g(z) \neq$ 0 , we have $\phi(z)=\phi(z)$. Since $\phi$ is an automorphism of $\mathbb{D}$ and $\phi(0)=0$, it follows that $\phi(z)=\lambda z$, where $|\lambda|=1$, that is $\phi$ is a rotation.

From the proof of the above theorem, it is easy to see that if $C_{\phi}$ is an isometry on $\mathcal{D}_{\alpha}^{p}, 1 \leq p<\infty$ then $\phi(0)=0$. We can get the following corollary.

Corollary 2.3. Let $\varphi \in \operatorname{Aut}(\mathbb{D})$. Then the induced composition operator $C_{\phi}$ is an isometry on the weighted Dirichlet-type space $\mathcal{D}_{\alpha}^{p}, 1 \leq p<\infty$ and $-1<\alpha<\infty$, if and only if $\phi$ is a rotation map.

Clearly, if both $C_{\phi}$ and $M_{u}$ are isometries, then $W_{u, \phi}$ is an isometry, the following theorem will show that the converse is also true.

Theorem 2.4. The weighted composition operator $W_{u, \phi}$ is an isometric operator on $\mathcal{D}_{\alpha}^{p}, 1 \leq p<\infty$ and $\alpha>-1$, if and only if both $C_{\phi}$ and $M_{u}$ are isometric operators.

Proof. Suppose $W_{u, \phi}$ is an isometry. Replacing $C_{\phi}$ by $W_{u, \phi}$ and using the same methods and the same assumption in Theorem 2.2, we get

$$
\left\|\left(W_{u, \varphi} 1\right)^{\prime}+t\left(W_{u, \varphi} p_{n}\right)^{\prime}\right\|_{A_{\alpha}^{p}}=\left\|\left(W_{u, \varphi} 1\right)^{\prime}\right\|_{A_{\alpha}^{p}}+|t|\left\|\left(W_{u, \varphi} p_{n}\right)^{\prime}\right\|_{A_{\alpha}^{p}}
$$

It follows from (2.2) that the real valued function

$$
p(t)=\left\|\left(W_{u, \varphi} 1\right)^{\prime}+t\left(W_{u, \varphi} p_{n}\right)^{\prime}\right\|_{A_{\alpha}^{p}}
$$

is not a differentiable function of $t$ at $t=0$. However, in the terminology of [19], the $L^{p}$ norm is weakly differentiable at every point except the zero vector, that is $\|\cdot\|_{A_{\alpha}^{p}}$ is not weakly differentiable at $\left(W_{u, \phi} 1\right)^{\prime}$. Consequently

$$
\left(W_{u, \varphi} 1\right)^{\prime}(z)=0
$$

for every $z \in \mathbb{D}$. So $\left(W_{u, \phi} 1\right)^{\prime} \equiv 0$, which implies that $u=W_{u, \phi} 1$ is a constant. 
Since $W_{u, \phi}$ is an isometry, $\|u\|_{\mathcal{D}_{\alpha}^{p}}=\left\|W_{u, \varphi} 1\right\|_{\mathcal{D}_{\alpha}^{p}}=\|1\|_{\mathcal{D}_{\alpha}^{p}}=1$, and consequently, $u \equiv$ $\lambda$, for some $|\lambda|=1$. Hence the multiplication operator $M_{u}$ is an isometry. Now,

$$
\|f\|_{\mathcal{D}_{\alpha}^{p}}=\left\|W_{u, \varphi} f\right\|_{\mathcal{D}_{\alpha}^{p}}=\left\|\left(u C_{\varphi}\right)(f)\right\|_{\mathcal{D}_{\alpha}^{p}}=\left\|\lambda C_{\varphi}(f)\right\|_{\mathcal{D}_{\alpha}^{p}}=\left\|C_{\varphi}(f)\right\|_{\mathcal{D}_{\alpha}^{p}}
$$

for every $f \in \mathcal{D}_{\alpha}^{p}$. Hence the composition operator $C_{\phi}$ is an isometry.

Remark. From the above the theorem, we can get that the multiplier operator $M_{u}$ is an isometric operator on $\mathcal{D}_{\alpha}^{p}, 1 \leq p<\infty,-1<\alpha<\infty$ if and only if $u$ is a constant of modulus one. In [20], Aleman et al. characterized the nontrivial isometric multipliers on the Dirichlet-type space $\mathcal{D}_{w}^{p}, 1 \leq p<\infty$ (here $w$ is the weighted function). For the sake of completeness, we state their results as follows.

Theorem 2.5. Let $1 \leq p<\infty$. Suppose that the Dirichlet-type space $\mathcal{D}_{w}^{p}$ is complete and that point-evaluations are bounded. Then $\mathcal{D}_{w}^{p}$ has nonconstant isometric pointwise multipliers if and only if $p=2$ and $w(z)=-2 \log |z|$ a.e. in $\mathbb{D}$. In this case $\mathcal{D}_{w}^{p}=H^{2}$ and the isometric multipliers are precisely the inner functions.

\section{Acknowledgements}

The authors would like to thank the referees for the useful comments and suggestions which improved the presentation of this article. ZHZ supported in part by the National Natural Science Foundation of China (Grant Nos. 10971153, 10671141)

\section{Author details}

${ }^{1}$ College of Mathematics and Statistics, Chongqing Technology and Business University, Chongqing 400067, People's Republic of China 'Department of Mathematics,Tianjin University, Tianjin 300072, People's Republic of China

\section{Authors' contributions}

All authors conceived and drafted the manuscript, and read and approved the final manuscript.

\section{Competing interests}

The authors declare that they have no competing interests.

Received: 12 April 2011 Accepted: 9 February 2012 Published: 9 February 2012

\section{References}

1. Hibschweiler, RA: Composition operators on Dirichlet-type spaces. Proc Am Math Soc. 128, 3579-3586 (2000). doi:10.1090/S0002-9939-00-05886-X

2. Roan, R: Composition operators on the space of functions with $H^{P}$-derivative. Houst J Math. 4, 423-438 (1978)

3. Martín, MJ, Vukotić, D: Isometries of some classical function spaces among the composition operators. Contemp Math. 393, 133-138 (2006)

4. Kolaski, CJ: Isometries of weighted Bergman spaces. Can J Math. 34(4):910-915 (1982). doi:10.4153/CJM-1982-063-5

5. Kolaski, CJ: Surjective isometries of weighted Bergman spaces. Proc Am Math Soc. 105, 652-657 (1989). doi:10.1090/ S0002-9939-1989-0953008-7

6. Carswell, BJ, Hammond, C: Composition operators with maximal norm on weighted Bergman spaces. Proc Am Math Soc. 134, 2599-2605 (2006). doi:10.1090/S0002-9939-06-08271-2

7. Martín, MJ, Vukotić, D: Isometries of the Bloch space among the composition operators. Bull Lond Math Soc. 39, 151-155 (2007)

8. Colonna, F: Characterisation of the isometric composition operators on the Bloch space. Bull Aust Math Soc. 72 283-290 (2005). doi:10.1017/S0004972700035073

9. Allen, RF, Colonna, F: On the isometric composition operators on the Bloch space in $\mathbb{C}^{n}$. J Math Anal Appl. 355 675-688 (2009). doi:10.1016/j.jmaa.2009.02.023

10. Allen, RF, Colonna, F: Isometries and spectra of multiplication operators on the Bloch space. Bull Aust Math Soc. 79 147-160 (2009). doi:10.1017/S0004972708001196

11. Li, GL, Zhou, ZH: Isometries on products of composition and integral operators on Bloch type space. J Inequal Appl. 2010, 8 (2010)

12. Cohen, JM, Colonna, F: Isometric composition operators on the Bloch space in the polydisk. Contemp Math. 454, 9-21 (2008)

13. Li, SY: Composition operators and isometries on holomorphic function spaces over domains in $\mathbb{C}^{n}$. AMS/IP Stud Adv Math. 39, 161-174 (2007)

14. Li, SY, Ruan, YB: On characterizations of isometries on function spaces. Sci China Ser A Math. 51(4):620-631 (2008). doi:10.1007/s11425-007-0145-X

15. Laitila, J: Isometric composition operators on BMOA. Math Nachr. 283, 1646-1653 (2010). doi:10.1002/mana.200710164 
16. Martín, MJ, Vukotić, D: Isometries of the Dirichlet space among the composition operators. Proc Am Math Soc. 134(6):1701-1705 (2006). doi:10.1090/50002-9939-05-08182-7

17. Novinger, W, Oberlin, D: Linear isometries of some normed spaces of analytic functions. Can J Math. 37, $62-76$ (1985). doi:10.4153/CJM-1985-005-3

18. Fleming, RJ, Jamison, JE: Isometries on Banach spaces: function spaces, vol. 129. CHAPMAN HALL/CRC Monographs and Surveys in Pure and Applied Mathematics. Boca Raton (2003)

19. Köthe, G: Topological vector spaces I. Springer, New York (1969)

20. Aleman, A, Duren, P, Martín, MJ, Vukotić, D: Multiplicative isometries and isometric zero-divisors. Can J Math. 62, 961-974 (2010). doi:10.4153/CJM-2010-048-7

doi:10.1186/1029-242X-2012-23

Cite this article as: Geng et al:: Isometric composition operators on weighted Dirichlet-type spaces. Journal of Inequalities and Applications 2012 2012:23.

\section{Submit your manuscript to a SpringerOpen ${ }^{\odot}$} journal and benefit from:

- Convenient online submission

- Rigorous peer review

- Immediate publication on acceptance

- Open access: articles freely available online

- High visibility within the field

- Retaining the copyright to your article

Submit your next manuscript at $\boldsymbol{\nabla}$ springeropen.com 